\title{
“Tu n'en as pas assez de frotter le carrelage des riches ? [...] Tu ne préférerais pas travailler avec nous dans la résistance?"
}

In memoriam Nelly Sturm, née Klein (1924-2017) et ses activités de résistante au sein du groupe communiste Travail allemand pendant l'occupation allemande de la Belgique

In memoriam: Nelly Sturm-Klein (1924-2017) en haar verzet binnen de Duitse communistische groep Travail allemand

\section{Tanja von Fransecky}

Traducteur : Emilie Syssau

\section{(2) OpenEdition}

\section{Journals}

Édition électronique

URL : https://journals.openedition.org/temoigner/7352

DOI : $10.4000 /$ temoigner.7352

ISSN : 2506-6390

Traduction(s) :

In memoriam: Nelly Sturm-Klein (1924-2017) en haar verzet binnen de Duitse communistische groep

Travail allemand - URL : https://journals.openedition.org/temoigner/7407 [nl]

Éditeur :

Éditions du Centre d'études et de documentation Mémoire d'Auschwitz, Éditions Kimé

\section{Édition imprimée}

Date de publication : 2 avril 2018

Pagination : 122-127

ISBN : 978-2-930953-06-9

ISSN : 2031-4183

\section{Référence électronique}

Tanja von Fransecky, «"Tu n'en as pas assez de frotter le carrelage des riches ? [...] Tu ne préférerais pas travailler avec nous dans la résistance ?" », Témoigner. Entre histoire et mémoire [En ligne], 126 | 2018, mis en ligne le 24 janvier 2022, consulté le 04 février 2022. URL : http://

journals.openedition.org/temoigner/7352 ; DOI : https://doi.org/10.4000/temoigner.7352 


\section{"Tu n'en as pas assez de frotter le carrelage des riches? [...] Tu ne préférerais pas travailler avec nous dans la Résistance? „}

In memoriam Nelly Sturm, née Klein (1924-2017)

et ses activités de résistante au sein du groupe

communiste Travail allemand pendant

l'occupation allemande de la Belgique'.

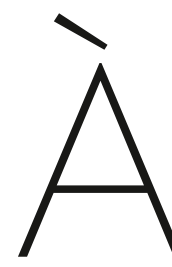

la suite de l'invasion de l'Autriche par la Wehrmachtet, en conséquence, la suite delinvasion delAutriche par la Wehrmachtet, enconséquence, sitôt à persécuter les opposants politiques et la population juive. Les Sitôt à persécuter les opposants politiques et la population juive. Les $\mathrm{SA}$, les SS, les Jeunesses hitlériennes et une partie de la population Juifs d'Autriche, une terreur rappelant les pogroms contre les quelque 200000 individus que comptait la population juive ; elle dura des mois et se solda par la fuite de nombre d'entre eux.

Nelly Klein, alors âgée de treize ans, fuit également Vienne avec ses parents, Elisabeth et Koloman Klein à la fin de l'été 1939, pour gagner la Belgique. À peine Elisabeth un an plus tard, le 10 Kai 1940, la Wehrmacht les y rattrape. Juste après l'entrée de l'armée allemande dans le pays, les autorités belges arrêtent près de 10000 migrant allemands et autrichiens, parmi lesquels de nombreux Juifs et opposants politiques aux nationaux-socialistes, soupçonnés d'être la « Cinquième colonne », à savoir des espions d'Hitler. Les ex-Autrichiens, citoyens allemands depuis l'annexion de l'Autriche en 1938, sont également concernés par ces arrestations. Sur la base d'une convention contraire au droit international passée entre les autorités belges et françaises, les hommes arrêtés sont déportés dans des camps d’internement déjà existants dans le sud de la France Ces camps avaientétéinitialement aménagés par le gouvernement français pour interner les membres des brigades internation par le goulternationale frappé par ce destin le 14 mai 1940, et emmené en France. Nelly et Elisabeth Klein

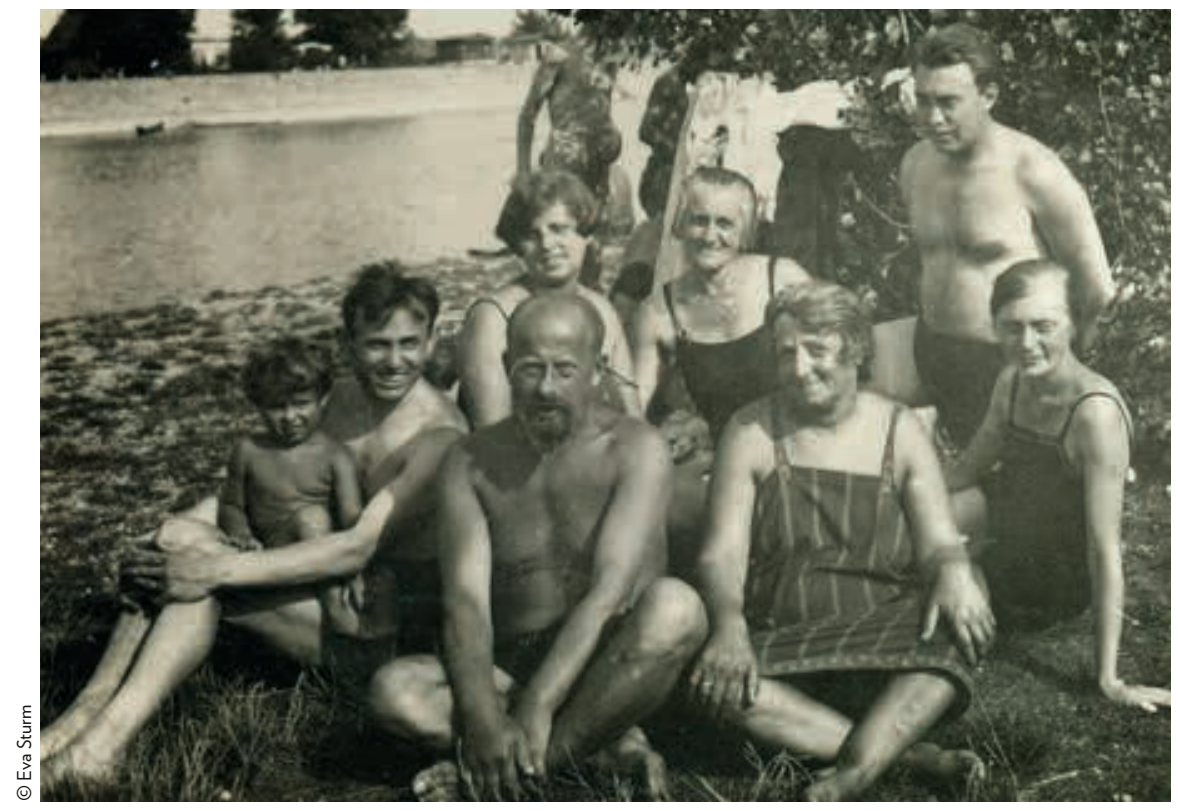

-Sortie balnéaire sur les à Kuchelau, été 1926; Nelly Klein, ses parents Elisabeth
et Koloman Klein et ses grands-parents maternel parviennent encore à lui apporter quelques affaires personnelles avant sa déportation au camp de Saint-Cyprien. S’ensuit alors une odyssée de près de deux ans, qui le conduit d'abord dans les camps d'internement de la zone (encore) inoccupée par la Wehrmacht dans le sud de la France : Saint-Cyprien, Gurs, Les Milles. Il est finalement déporté à Auschwitz le 17 août 1942, au départ de la gare de Drancy-Le Bourget. Ses proches n'entendront plus jamais parler de lui à partir de sa déportation dans le sud de la France.

Comme dans tous les pays sous occupation allemande, la persécution et l'assassinat de la population juive de Belgique sont des objectifs prioritaires des occupants. Pour le men a bi no ils Pour et le Conseil juif au service du commandant, de la police de sécurité et du SD. Le 4 août 1942, le premier train de déportés quitte le camp de regroupement de Malines vers Auschwitz, tandis que l'on pourchasse la population juive lors de rafles.

Nelly et sa mère se fondent dans la clandestinité comme aides ménagères. Être aide ménagère clandestine signifie être entièrement livrée à l'humeur de ses employeurs - il n'est pas rare que ceux-ci aient recours à la dénonciation. À l'automne 1942, Nelly travaille chez un couple très âgé à l'ouest de Bruxelles. Alors qu'elle nettoie la facade carrelée de la maison au balai-brosse Judith Fürst une amie de l'ancien groupe de jeunes du Comité juif passe par là et lui demande : "Tu n'en as pas a as pas assez de fotter laviller 
lequel recrute Judith Fürst s’appelle Travail allemand (TA). L’idée d'exploiter pour la Résistance les connaissances en allemand des communistes exilés voit le jour à l'été 1941, au sein du Parti communiste français (PCF), à une époque où, à la suite de l'attaque de l'Union soviétique par l'Allemagne et de la rupture du pacte RibbentropMolotov, on discute des possibilités de résister aux occupants allemands. Créées en décembre 1941 à Paris à l'instigation du PCF par des fonctionnaires des partis communistes autrichien, allemand et tchèque, des factions du groupe TA apparteallemande » du Parti communiste allemand en exil.

Si les groupes de résistants belges reçoivent de l'argent et des armes via l'Angleterre, ce n'est pas le cas des groupes autrichiens ou allemands. En Belgique, le TA est certes en contact avec d'autres groupes de résistants belges, mais ceux-ci ne pensent visiblement pas à leur remettre des armes, par ailleurs en nombre restreint: à leurs yeux, ils restent des Allemands ou des Autrichiens, et leur semblent à ce titre peu dignes de confiance. Les jeunes gens sont donc livrés à eux-mêmes et misent essentiellement sur des formes de résistance qui profiteraient de leurs connaissances en allemand, leur langue maternelle. Une des formes de résistance est toutefois effectuće exclusivenent par de jeunes femmes : le Müdelarbeit ou Soldatenarbeit.

\section{LE MÄDELARBEIT OU SOLDATENARBEIT DU TA}

Nelly Klein fait bientôt partie de la vingtaine de femmes dotées de faux papiers originaires pour la plupart d'Autriche, mais aussi pour quelques-unes d'Allemagne de Russie et de Pologne, essentiellement issues de familles juives assimilées. Certaines d'entre elles ont déjà fait partie d'organisations de jeunesse de gauche avant leur émigration

Elles doivent séduire les soldats de la Wehrmacht stationnés en Belgique. Les juives et comor pue des lieux publics pour évitent souvent par doux et rencold dan contact es la pour éviter tout harcèlement éventuel. Le but de cette prise de contact est la subversion de la force armée. Les jeunes femmes doivent ainsi, en conversant avec les soldats, semer le doute sur la guerre, et surtout sur la « Solution finale », dans l'espoir de les amener à déserter. Elles doivent en outre solliciter des explications et exercer un espionnage militaire. Elles essaient de se renseigner sur les déplacements prévus des troupes, sur l'humeur des soldats ainsi que sur leurs peurs et leurs inquiétudes. Elles doivent gagner à leur cause les soldats de la Wehrmacht, afin de les amener à répandre dans les casernes une attitude critique enver la guerre, transmettre des informations internes, passer des tracts au-delà der la guerre, transinet oindre les partisans slovènes de Carinthie ou plus tard encore les troupes alliées.
Elles rencontrent les soldats entre autres sur les marchés aux puces, où elles se font passer pour des interprètes quand elles remarquent des difficultés linguistiques. En général, les soldats apprécient de rencontrer des jeunes filles et des jeunes femmes avec qui ils peuvent parler allemand, et il arrive souvent que ces rencontres « de hasard » se terminent devant un café. À la première rencontre, les jeunes filles doivent éviter les discussions sur le déroulement de la guerre et la politique, pour d'abord sympathiser avec les soldats sur un plan personnel. Ce n'est qu’à la rencontre suivante que, dans le cadre du renseignement militaire, elles doivent rencon comment. Mais es soldats, gênerars Après la dissolution du Komintern en mai 1943 par Staline, qui appelle alors tous les pays à représenter leurs intérêts nationaux sans influence étrangère, les jeunes femmes s'adressent exclusivement à des soldats de la Wehrmacht autrichiens. La lutte pour l'autonomie nationale de l'Autriche prend encore plus d'importance au sein du groupe autrichien du TA, visiblement avivée par l'attitude chauvine de certains fonctionnaires du parti communiste allemand, ainsi que par la déclaration de Moscou du $1^{\text {er }}$ novembre 1943, qui exige une contribution autonome des Autrichiens à la libération de leur pays. Carte d'identité falsifiée Klein
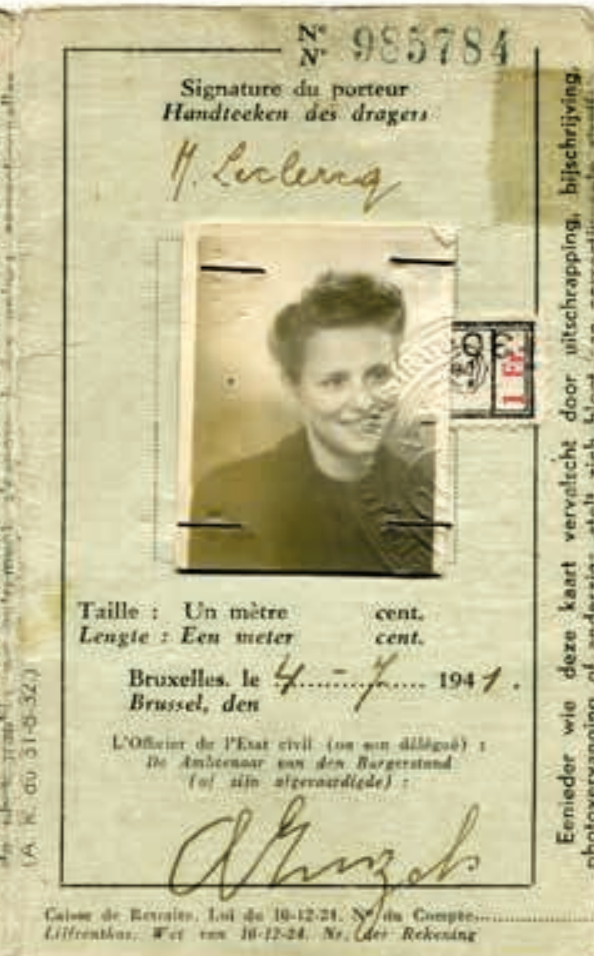

$-N^{N} 995784$
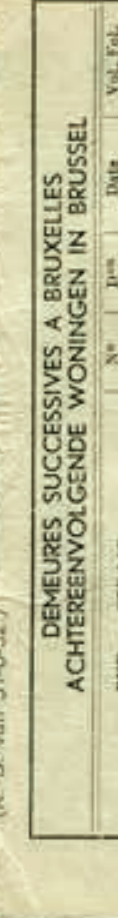
DES RTHU DANS LA RÉSISTANCE? - Nelly Klein peu après de quelques camarades. Rangée supérieure,
de gauche à droite: de gauche a droite:
probablement Alfred
Wiesinger, probableme Helene Meyersohn nconnu, Nelly Klein, ranconnué inférieure Krochmal; à droite : Cilly Spitz, Hertha

Rares sont les soldats que Nelly Klein rencontre plus d'une ou deux fois, ces contacts se révélant sans suite. D’après elle, la plupart des jeunes soldats croient fermement à la victoire finale

Je me rappelle de leur dévouement au Führer et de leur exaltation.

De la discipline, vertu suprême. Je me souviens qu'ils n’avaient pas la moindre compassion pour les peuples opprimés [.... La propagande nazie avait fait des dégâts inoüs dans la

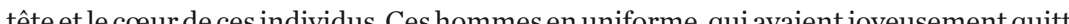
leurs villages et petites vilt arrogance incroyable.

Il n'est pas facile d'identifier parmi eux les adversaires de la guerre et des nazis. Les jeunes femmes rencontrent tantôt des nationaux-socialistes convaincus, tantôt

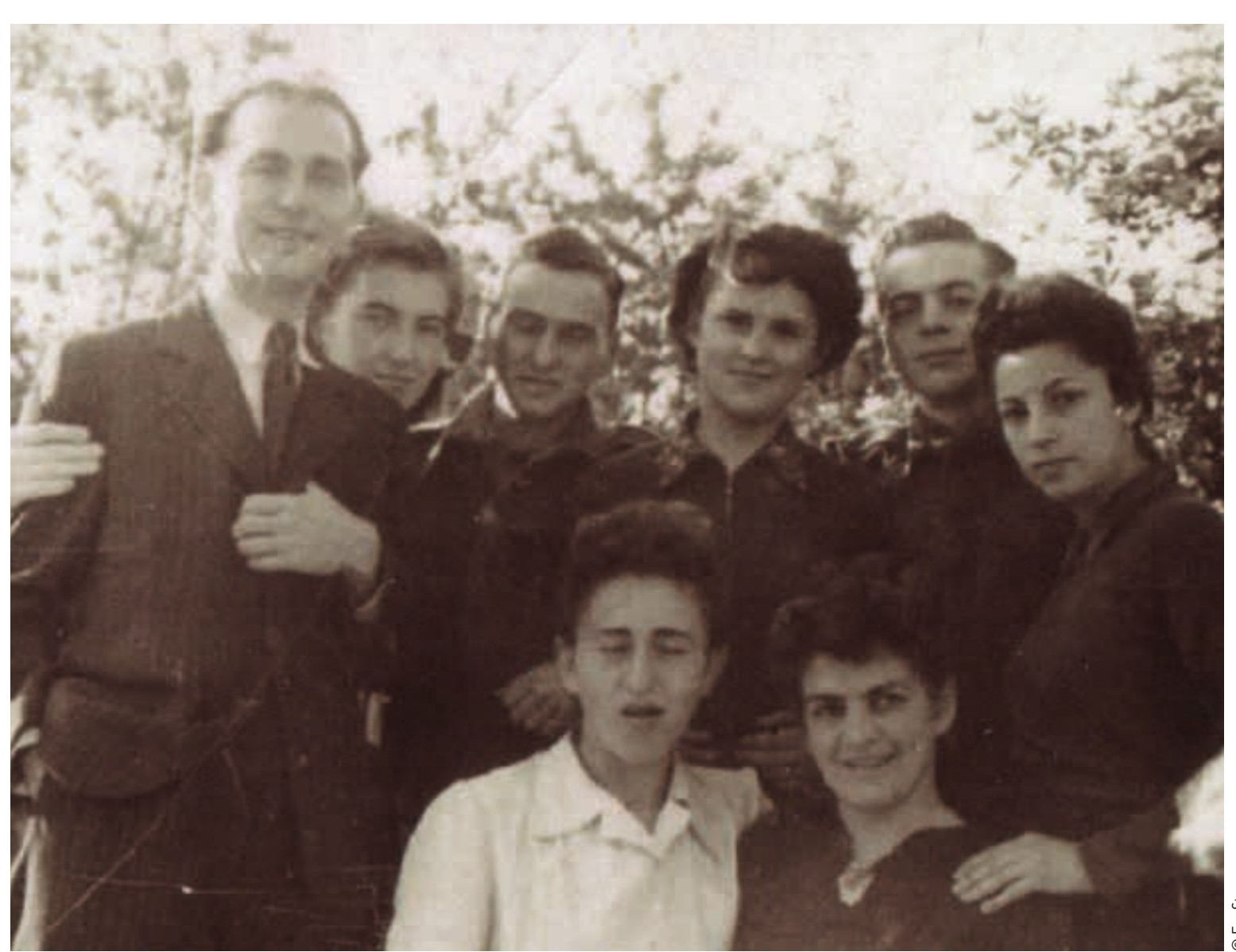

des sceptiques. Certains fanfaronnent avec des photos de partisans pendus, mais quelques-uns disent : « Si les Russes viennent à nous - après les atrocités inimaginables que nous avons commises chez eux - il ne restera pas un édifice debout. » Nelly Sturm explique ce que représentaient pour elle les tentatives d'amener les soldats à refuser d'obéir :

Mis à part ma peur lors de ces rencontres, ce travail a toujours eu pour moi un côté fas-

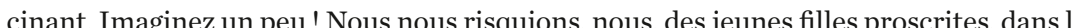
cinant. Imaginez un per gueule du loup, pour voir ce qu'il avait dans le ventre... Et ensuite, nous les appelions auss à se révolter contre leurs superieurs! Je n'oubliais janas, an moment de les quitter, de leur dire avec un doux sourire : « Vous ne pouvez pas gagner cette guerre. Vous allez la perdre. Ce serait donc mieux d'arrêter tout de suite.»

Quelques soldats de la Wehrmacht désertent effectivement avec le soutien de jeunes Autrichiennes. A l'été 1942, on parvient même à prendre contact avec les soldats du bataillon pénitentiaire 999 qui doit embarquer à Anvers pour l'Afrique du Nord. Les soldats de ce bataillon étaient souvent des opposants politiques au national-socialisme, recrutés de forcedans les prisons ou les camps de concentration pour des opérations militaires dont on savait quelles subiraient de lourdes pertes.

Les pertes dans les rangs du TA furent très élevées. Lors desformations, les soldats de la Wehrmacht étaient mis en garde contre les «femmes bolchéviques qui s'expri-
maient en allemand.» Bien des jeunes femmes payèrent de leur vie leur implication, maient en allemand.» Bien des jeunes femmes payèrent de leur vie leur implication, ce titre, punie de mort. D'autres ontété durement torturées et déportées à Auschwitz. Nelly Klein a échappé à la déportation, mais ses parents ont été assassinés. La mère de Nelly a connu un destin terrible. Le SS-Hauptsturmführer Prof. Dr. August Hirt, directeur de l'Institut d'anatomie à l'Université de Strasbourg, prévoyait d'assassiner des détenus juifs de camps de concentration dans la chambre à gaz du camp de Natzweiler-Struthof Leurs cadavres étudiés à l'Institut d'anatomie de l'Unive de , sous forme de squelette, co de la science raciale national-socialiste, destinée à la recherche anthropologique. Les chercheurs des générations futures auraient ainsi eux aussi la possibilité d’étudier les «caractéristiques raciales » des Juifs, même après leur extermination. Elisabeth Klein se trouvait parmi les Juifs d'Auschwitz dont August Hirt avait demandé la livraison au camp de concentration de Natzweiler-Struthof à ces fins.

Nelly Klein a vécu la Libération à Bruxelles. Après une vie riche d'engagements politiques, émaillée d'étapes en Belgique, Autriche, RDA, France et RFA, elle est décédée le 9 mars 2017 à lâge de 92 ans à Berlin. I

Tania von Fransecky est chercheuse au département de la «persécution des Juifs» aux Archives nationales d'Allemagne (Bundesarchiv). 